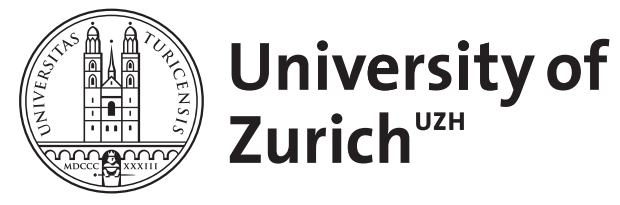

Zurich Open Repository and Archive

University of Zurich

University Library

Strickhofstrasse 39

CH-8057 Zurich

www.zora.uzh.ch

Year: 2006

\title{
A refined finite element convergence theory for highly indefinite Helmholtz
} problems

Sauter, S A

DOI: https://doi.org/10.1007/s00607-006-0177-z

Posted at the Zurich Open Repository and Archive, University of Zurich ZORA URL: https://doi.org/10.5167/uzh-21640

Journal Article

Accepted Version

Originally published at:

Sauter, S A (2006). A refined finite element convergence theory for highly indefinite Helmholtz problems.

Computing, 78(2):101-115.

DOI: https://doi.org/10.1007/s00607-006-0177-z 


\title{
A Refined Finite Element Convergence Theory for Highly Indefinite Helmholtz Problems
}

\author{
S. Sauter* \\ Dedicated to Prof. Dr. Ivo Babuška on the occasion of his 80th birthday
}

\begin{abstract}
It is well known that standard $h$-version finite element discretisations for Helmholtz' equation suffer from the following stability condition: "The mesh width $h$ of the finite element mesh has to satisfy $k^{2} h \lesssim 1$ ", where $k$ denotes the wave number. This condition rules out the reliable numerical solution of Helmholtz equation in three dimensions for large wave numbers $k \gtrsim 50$.

In our paper, we will present a refined finite element theory for highly indefinite Helmholtz problems where the stability of the discretisation can be checked through an "almost invariance" condition.

As an application, we will consider a one-dimensional finite element space for the Helmholtz equation and apply our theory to prove stability under the weakened condition $h k \lesssim 1$ and optimal convergence estimates.
\end{abstract}

\section{Introduction}

In this paper, we will consider the numerical solution of the Helmholtz equation by the finite element method. Let $\Omega \subset \mathbb{R}^{d}$ be a bounded Lipschitz domain with boundary $\Gamma$. The Helmholtz problem in the classical form with Robin boundary conditions is given by

$$
\begin{array}{cc}
-\Delta u-k^{2} u=f & \text { in } \Omega, \\
\frac{\partial u}{\partial n}+\mathrm{i} k u=g & \text { on } \partial \Omega,
\end{array}
$$

where we assume throughout the paper that the wave number is positive and bounded away from zero, i.e., $k \geq k_{0}>0$.

The variational form is given by seeking $u \in H^{1}(\Omega)$ such that

$$
a(u, v):=\int_{\Omega}\langle\nabla u, \nabla \bar{v}\rangle-k^{2} u \bar{v}+\mathrm{i} k \int_{\Gamma} u \bar{v}=\int_{\Omega} f \bar{v}+\int_{\Gamma} g \bar{v}=: F(v) \quad \forall v \in H^{1}(\Omega) .
$$

We equip the space $H^{1}(\Omega)$ with the norm

$$
\|u\|_{\mathcal{H}}:=\left(|u|_{1, \Omega}^{2}+k^{2}\|u\|_{0, \Omega}^{2}\right)^{1 / 2}
$$

which is obviously equivalent to the $H^{1}$-norm.

For Lipschitz domains, it is well known that a trace estimate holds.

*(stas@amath.unizh.ch), Institut für Mathematik, Universität Zürich, Winterthurerstr 190, CH-8057 Zürich, Switzerland 
Lemma 1.1 There exists a constant $C_{\mathrm{tr}}$ depending only on $\Omega$ and $k_{0}$ such that

$$
\forall u \in H^{1 / 2}(\partial \Omega): \quad\|u\|_{H^{1 / 2}(\partial \Omega)} \leq C_{\text {tr }}\|u\|_{\mathcal{H}} .
$$

Theorem 1.2 Let $\Omega$ be a bounded Lipschitz domain. Then, there is a constant $C(\Omega, k)>0$ such that for all $f \in\left(H^{1}(\Omega)\right)^{\prime}, g \in H^{-1 / 2}(\Gamma)$, the unique solution u of problem (1.2) satisfies

$$
\|u\|_{\mathcal{H}} \leq C(\Omega, k)\left(\|f\|_{H^{1}(\Omega)^{\prime}}+\|g\|_{H^{-1 / 2}(\Gamma)}\right) .
$$

For a proof, we refer to [15, Proposition 8.1.3].

The explicit dependence of the constant $C(\Omega, k)$ on the wave number is more subtle to derive. The following proposition is taken from [15, Proposition 8.1.4].

Proposition 1.3 Let $\Omega$ be a bounded star-shaped domain with smooth boundary or a bounded convex domain. Then, there is $C_{\mathrm{reg}}>0$ (depending only on $\Omega$ ) such that for any $f \in L^{2}(\Omega)$, $g \in H^{1 / 2}(\partial \Omega)$, the solution of the Helmholtz problem satisfies

$$
\begin{aligned}
\|u\|_{\mathcal{H}} & \leq C_{\text {reg }}\left(\|f\|_{L^{2}(\Omega)}+\|g\|_{L^{2}(\partial \Omega)}\right), \\
|u|_{H^{2}(\Omega)} & \leq C_{\text {reg }}\left\{C_{f, g} k+\|g\|_{H^{1 / 2}(\partial \Omega)}\right\}
\end{aligned}
$$

with

$$
C_{f, g}:=\|f\|_{L^{2}(\Omega)}+\|g\|_{L^{2}(\partial \Omega)} .
$$

Furthermore, we will need an estimate for the continuity constant.

Theorem 1.4 Let $\Omega$ be a bounded Lipschitz domain. Then, there exists $C_{c}>0$ depending only on $\Omega$ such that for all $u, v \in H^{1}(\Omega)$

$$
|B(u, v)| \leq C_{c}\|u\|_{\mathcal{H}}\|v\|_{\mathcal{H}} .
$$

The proof can be found in [15, Lemma 8.1.6].

This problem can be numerically solved by the finite element method. We recall here a convergence estimate for finite elements. Let $S \subset H^{1}(\Omega)$ be the space of continuous, piecewise linear finite elements on a regular finite element mesh $\mathcal{G}$ with maximal step size $h$ and $u_{S}$ the corresponding Galerkin solution. We assume the approximation property for piecewise linear finite elements: There exists a constant $C_{\text {ap }}$ depending only on $\Omega$ and the minimal angles in the triangulation such that, for all $v \in H^{2}(\Omega)$, there holds

$$
\inf _{v \in S}\left(\|u-v\|_{L^{2}(\Omega)}+h|u-v|_{H^{1}(\Omega)}\right) \leq C_{\mathrm{ap}} h^{2}\left(|v|_{H^{2}(\Omega)}+k\|v\|_{\mathcal{H}}\right) .
$$

Theorem 1.5 Let $\Omega$ be a bounded star-shaped domain with smooth boundary (or a bounded convex domain). Then, there exist positive constants $C_{1}, C_{2}, C_{3}$ depending only on $\Omega$ and the angles of the triangulation so that, under the assumption $\left(1+k^{2}\right) h<C_{1}$, there holds

$$
\inf _{u \in S} \sup _{v \in S} \frac{\operatorname{Re} a(u, v)}{\|u\|_{\mathcal{H}}\|v\|_{\mathcal{H}}} \geq \frac{C_{2}}{1+k} .
$$

The finite element solution $u_{S}$ satisfies

$$
\left\|u-u_{S}\right\|_{\mathcal{H}} \leq C_{3} \inf _{v \in S}\|u-v\|_{\mathcal{H}} \leq C C_{3} C_{\mathrm{ap}} C_{\mathrm{reg}} h k\left(\|g\|_{H^{1 / 2}(\partial \Omega)}+\|f\|_{L^{2}(\Omega)}\right),
$$

where $C$ only depends on $k_{0}$. 
Proof. The proof follows by combining [15, Proposition 8.2.7], Theorem 1.3, and (1.3).

This behavior of the finite element error is rather unsatisfactory since the stability condition on the mesh width, namely " $k^{2} h$ is sufficiently small", is quite strong.

In [4], a generalized finite element method was presented in one dimension, where the stability " $k k^{2} h \lesssim 1$ is relaxed to " $k h \lesssim 1$ ". Explicit basis functions for this finite element method have been presented in [12]. However, the proofs in these papers rely on the explicit knowledge of the discrete Green's function and, hence, do not carry over to higher dimensions.

On the other hand, the general stability and convergence analysis which was developed in [15] do not yield improved estimates when applied to the generalized finite element method.

In this paper, we will generalize the theory in [15] to yield optimal error estimates for generalized finite element methods for the Helmholtz problem. As an important side effect, this theory can be used as a guideline for the construction of modified finite element methods for the Helmholtz problem since it links the discrete stability and convergence of the Galerkin discretization to an "almost invariance property" of the finite element space which can be stated qualitatively as follows:

"If the right-hand side of the Helmholtz problem is a function in the (modified) finite element space, then, the corresponding continuous Helmholtz solution must lie "almost" in the (modified) finite element space itself (in the sense that a certain approximation property holds)."

More formally, let $S$ denote a (modified) finite element space and let $\mathcal{T}_{k}$ denote the solution operator for the continuous (adjoint) Helmholtz problem. Then, the space $S$ should satisfy

$$
\mathcal{T}_{k} S \approx S
$$

We call this property an "almost invariance property" and will specify the precise meaning of "ح" in this paper.

The literature on the development of high order and special finite elements for Helmholtz' problems at high wave number is vast, see, e.g., [6], [8], [9], [10], [16], [2]. In most cases, the goal of these approaches is to reduce the dispersion error, resp. the pollution effect of the finite element discretization when approximating waves. However, a general convergence analysis in $2 \mathrm{D}$ and $3 \mathrm{D}$ (which is explicit in the wave number and the mesh size/polynomial degree) is missing in most cases for these new types of finite elements. (For one-dimensional studies see, e.g., [12], [13], [14], [4].) Instead numerical comparisons are performed or a quantitative dispersion analysis is carried out, see, e.g. [1], [3], [6], [7], [8], [9], [11], [14], [16], [2], [5]. In contrast to these techniques, the goal of our paper is to provide a theory which can serve as the basis for the convergence analysis of new finite elements for the Helmholtz equation.

\section{Stability and Convergence Theory}

\subsection{Discrete Stability}

In this section, we will develop a theory where the stability of Galerkin's method can be formulated in terms of an "almost invariance property" of a finite element space.

Let $S$ denote a finite-dimensional subspace of $H^{1}(\Omega)$ and we assume always that the approximation property (1.3) holds. In this section, we will derive a condition for the space $S$ such that the discrete inf-sup constant can be bounded away from zero. 
Let $u \in S$ and put $v=u+z$, where $z \in H^{1}(\Omega)$ will be chosen later. Then,

$$
a(u, v)=a(u, u)+a(u, z)=\|u\|_{\mathcal{H}}+a(u, z)-2 k^{2}\|u\|_{0}^{2}+\mathrm{i} k \int_{\Gamma}|u|^{2} .
$$

Choose $z$ as the unique solution of:

$$
a(v, z)=2 k^{2}(u, v)-\mathrm{i} k \int_{\Gamma} u \bar{v} \quad \forall v \in H^{1}(\Omega) .
$$

We choose $v=u$ as a test function in (2.2) and, hence, equation (2.1) simplifies to

$$
a(u, v)=\|u\|_{\mathcal{H}}^{2} .
$$

Let $\mathcal{T}_{k}: S \rightarrow H^{1}(\Omega)$ denote the solution operator to problem (2.2), i.e.,

$$
z=\mathcal{T}_{k}(u)
$$

Define the space

$$
\mathcal{T}_{k}(S)=: \check{S} \subset H^{1}(\Omega) .
$$

We need an approximation property for the space $\check{S}$. Let

$$
\eta(S):=\sup _{u \in S \backslash\{0\}} \inf _{v \in S} \frac{\left\|\mathcal{T}_{k}(u)-v\right\|_{\mathcal{H}}}{\|u\|_{\mathcal{H}}} \text { and assume } \eta(S) \rightarrow 0 \text { as } \operatorname{dim} S \rightarrow \infty .
$$

Remark 2.1 The quantity $\eta(S)$ measures the "almost invariance" of the space $S$ under the solution operator $\mathcal{T}_{k}$ of the adjoint Helmholtz problem. The space $S$ would be invariant under $\mathcal{T}_{k}$ if and only if $\eta(S)=0$. It turns out that the construction of finite dimensional spaces $S$ with $\eta(S)=0$ is too complicated and the "almost invariance condition"

$$
\eta(S) \lesssim 1
$$

which we will be derived later (cf. (2.4)) gives much more flexibility in the construction of the finite element spaces.

For $u \in S$, let $z=\mathcal{T}_{k}(u)$ and denote by $z_{S} \in S$ the best approximation of $z$ with respect to the $\mathcal{H}$-norm. Then,

$$
\begin{aligned}
\left|a\left(u, u+z_{S}\right)\right| & \geq|a(u, u+z)|-\left|a\left(u, z-z_{S}\right)\right| \geq\|u\|_{\mathcal{H}}^{2}-C_{c}\|u\|_{\mathcal{H}}\left\|z-z_{S}\right\|_{\mathcal{H}} \\
& \geq\|u\|_{\mathcal{H}}^{2}-C_{c} \eta(S)\|u\|_{\mathcal{H}}^{2} .
\end{aligned}
$$

Choose $S$ with $\operatorname{dim} S$ sufficiently large that $C_{c} \eta(S)<\frac{1}{2}$. Then,

$$
\left|a\left(u, u+z_{S}\right)\right| \geq \frac{1}{2}\|u\|_{\mathcal{H}}^{2} .
$$

Note that Proposition 1.3 implies

$$
\|z\|_{\mathcal{H}} \leq C_{\mathrm{reg}}\left(\left\|k^{2} u\right\|_{L^{2}(\Omega)}+\|k u\|_{L^{2}(\partial \Omega)}\right) \leq C_{3} k\|u\|_{\mathcal{H}}
$$

with $C_{3}=C_{\text {reg }}\left(1+C_{\text {tr }}\right)$. Finally,

$$
\left\|u+z_{S}\right\|_{\mathcal{H}} \leq\|u\|_{\mathcal{H}}+\|z\|_{\mathcal{H}}+\left\|z-z_{S}\right\|_{\mathcal{H}} \leq\left(1+C_{3} k+C_{c} \eta(S)\right)\|u\|_{\mathcal{H}} .
$$

In summary, we have proved that the approximation property

$$
C_{c} \eta(S)<\frac{1}{2}
$$

leads to the discrete stability estimate of the finite element solution. 
Theorem 2.2 Let the assumptions of Proposition 1.3 be valid. Assume that the space $S$ satisfies

$$
C_{c} \eta(S)<\frac{1}{2}
$$

Then, the discrete inf-sup constant can be estimated by

$$
\inf _{u \in S} \sup _{v \in S} \frac{|a(u, v)|}{\|u\|_{\mathcal{H}}\|v\|_{\mathcal{H}}} \geq \frac{1}{\left(3+2 C_{3} k\right)} .
$$

Remark 2.3 In the standard case of linear finite elements $S_{h, 1}^{0}$, one can prove (cf. [15, Proof of Prop. 8.2.7])

$$
\eta(S) \leq \operatorname{ch} k^{2}
$$

which, in combination with (2.4), leads to the very restrictive condition on the mesh width: $h k^{2} \lesssim 1$

Remark 2.4 The measure of almost invariance gives guidelines for the construction of generalized finite element spaces: If the space $S$ satisfies $\eta(S) \leq$ chk, the stability of the Galerkin discretization can be proved under the condition $h k \lesssim 1$.

\subsection{Convergence Analysis}

In this section, we will prove the convergence of Galerkin's method. We assume throughout this section that the assumptions of Proposition 1.3 hold and the stability condition (2.4) is satisfied.

In the first step, we will estimate the $L^{2}$-error by the $H^{1}$-error and employ the AubinNitsche technique. The Galerkin error is denoted by $e=u-u_{S}$.

Let $\psi \in H^{1}(\Omega)$ be the unique solution of the adjoint problem

$$
a(v, \psi)=(e, v)_{L^{2}(\Omega)} \quad \forall v \in H^{1}(\Omega) .
$$

Let $\mathcal{S}_{k}: L^{2}(\Omega) \rightarrow H^{1}(\Omega)$ denote the solution operator to this problem, i.e., $\psi=\mathcal{S}_{k} e$, and define

$$
\tilde{\eta}(S):=\sup _{w \in L^{2}(\Omega) \backslash\{0\}} \inf _{v \in S} \frac{\left\|\mathcal{S}_{k} w-v\right\|_{\mathcal{H}}}{\|w\|_{L^{2}(\Omega)}} .
$$

We assume that $\tilde{\eta}(S) \rightarrow 0$ as $\operatorname{dim} S \rightarrow \infty$. Let $\psi_{S}$ denote the best approximation of $\psi$ in

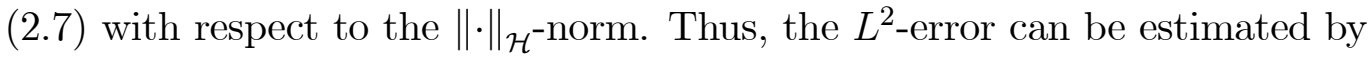

$$
\begin{aligned}
\|e\|_{L^{2}(\Omega)}^{2} & =a(e, \psi) \leq a\left(e, \psi-\psi_{S}\right) \leq C_{c}\|e\|_{\mathcal{H}}\left\|\psi-\psi_{S}\right\|_{\mathcal{H}} \\
& \leq C_{c} \tilde{\eta}(S)\|e\|_{\mathcal{H}}\|e\|_{L^{2}(\Omega)} .
\end{aligned}
$$

By using a Cauchy inequality for some $\varepsilon>0$, we obtain

$$
\|e\|_{L^{2}(\Omega)}^{2} \leq \frac{C_{c} \tilde{\eta}(S)}{2}\left(\varepsilon\|e\|_{L^{2}(\Omega)}^{2}+\frac{\|e\|_{\mathcal{H}}^{2}}{\varepsilon}\right) .
$$

The choice $\varepsilon=\frac{1}{C_{c} \tilde{\eta}(S)}$ finishes the proof of the auxiliary $L^{2}$-estimate:

$$
\|e\|_{L^{2}(\Omega)} \leq C_{c} \tilde{\eta}(S)\|e\|_{\mathcal{H}}
$$


To estimate the $\mathcal{H}$-norm of the error we proceed as follows. For any $v_{S} \in S$, it holds

$$
\begin{aligned}
\|e\|_{\mathcal{H}}^{2} & =\operatorname{Re}(a(e, e))+\left\{\|e\|_{\mathcal{H}}^{2}-\operatorname{Re} a(e, e)\right\} \\
& =\operatorname{Re} a\left(e, u-v_{S}\right)+2 k^{2}\|e\|_{L^{2}(\Omega)}^{2} \stackrel{(2.9)}{\leq} C_{c}\|e\|_{\mathcal{H}}\left\|u-v_{S}\right\|_{\mathcal{H}}+2\left(k C_{c} \tilde{\eta}(S)\right)^{2}\|e\|_{\mathcal{H}}^{2} .
\end{aligned}
$$

Now, we choose $S$ with $\operatorname{dim} S$ sufficiently large that

$$
2\left(k C_{c} \tilde{\eta}(S)\right)^{2} \leq \frac{1}{2} .
$$

Then, we arrive at the final estimate

$$
\|e\|_{\mathcal{H}} \leq 2 C_{c}\left\|u-v_{S}\right\|_{\mathcal{H}}
$$

Theorem 2.5 Assume that conditions (2.4) and (2.10) are satisfied and that the assumptions of Proposition 1.3 hold.

Then

$$
\|e\|_{\mathcal{H}} \leq 2 C_{c} \inf _{v \in S}\|u-v\|_{\mathcal{H}} .
$$

The $L^{2}$-error satisfies

$$
\|e\|_{L^{2}(\Omega)} \leq C_{c} \tilde{\eta}(S)\|e\|_{\mathcal{H}} .
$$

Remark 2.6 The error estimate (2.11) shows that the standard Galerkin error estimate for coercive bilinear forms, i.e.,

$$
\|e\|_{\mathcal{H}} \leq\left(1+\frac{C_{c}}{\gamma}\right) \inf _{v \in S}\|u-v\|_{\mathcal{H}}
$$

is too pessimistic since the quotient of the continuity constant $C_{c}$ and the discrete inf-sup constant $\gamma$ grows linearly in $k$ (cf. (2.5) and Theorem 1.4),

$$
C_{c} / \gamma \leq\left(3+2 C_{3} k\right) C_{c},
$$

where $C_{c}$ and $C_{3}$ are independent of $k$.

Remark 2.7 In the standard case of linear finite elements $S_{h, 1}^{0}$, one can prove (cf. [15, Proof of Prop. 8.2.7]

$$
\tilde{\eta}(S) \leq C h k
$$

which, in combination with (2.10), leads to the very restrictive condition on the mesh width: $h k^{2} \lesssim 1$.

For a modified finite element space the estimates (2.6), (2.12) can be improved.

\section{Application to a One-Dimensional Modified Finite El- ement Space}

In this section, we will consider a one-dimensional modified finite element space and analyze its stability and convergence properties by using the theory of the previous section. 


\subsection{The Method}

We define the finite element space for the Helmholtz problem as the span of some basis functions which, on each element satisfy the homogeneous Helmholtz equation. We restrict here to the one-dimensional case. Let $\Omega=(-1,1)$ and let $\mathcal{G}=\left(\tau_{m}: 1 \leq m \leq n\right)$ denote a partitioning of $\Omega$ into intervals $\tau_{m}:=\left(x_{m-1}, x_{m}\right)$. The mesh points satisfy

$$
-1=x_{0}<x_{1}<\ldots<x_{n-1}<x_{n}=1 .
$$

The basis functions $b_{m}$ are defined by (cf. [12])

$$
\forall 0 \leq m \leq n: \quad b_{m}(x):=\left\{\begin{array}{cl}
\frac{\sin k\left(x-x_{m-1}\right)}{\sin k\left(x_{m}-x_{m-1}\right)} & \text { if } x \in \tau_{m} \text { and } m \geq 1, \\
\frac{\sin k\left(x-x_{m+1}\right)}{\sin k\left(x_{m}-x_{m+1}\right)} & \text { if } x \in \tau_{m+1} \text { and } m<n, \\
0 & \text { otherwise. }
\end{array}\right.
$$

Note that these basis functions satisfy the homogeneous Helmholtz equation in every interval $\tau \in \mathcal{G}$. Furthermore, the function $b_{m}$ has value 1 in $x_{m}$ and vanishes at all other grid points. Finally, the support of the basis function is the same as the support of the usual piecewise linear hat functions.

Let $S:=\operatorname{span}\left\{b_{i}: 0 \leq i \leq n\right\}$. The Galerkin method for problem (1.2) is given by seeking $u \in S$ such that

$$
a(u, v)=F(v) \quad \forall v \in S .
$$

Theorem 3.1 Let the assumptions of Proposition 1.3 be satisfied. There exists constants $\alpha_{0}>0$ and $C$ independent of $k \geq k_{0}$ such that the Galerkin solution exists for all $k h<\alpha_{0}$ and satisfies the error estimates

$$
\left\|u-u_{S}\right\|_{\mathcal{H}} \leq C h \quad \text { and } \quad\left\|u-u_{S}\right\|_{L^{2}(\Omega)} \leq C h^{2} k .
$$

The constant $C$ depends on the data $f$ and $g$. by

Proof. We will estimate $\eta(S), \eta(\tilde{S})$ and the interpolation error in the remaining sections

$$
\eta(S) \leq C k h, \quad \eta(\tilde{S}) \leq C h, \quad\left\|u-u^{\mathrm{int}}\right\|_{\mathcal{H}} \leq C h .
$$

The combination with Theorem 2.2 and Theorem 2.5 yields the proof.

\subsection{Estimate of $\eta(S)$}

We start with the estimate of the quantity $\eta(S)$ as defined in (2.3). The basic tool will be the mapping behavior of the operator $\mathcal{I}_{k}$ on the space $S$. The strong formulation of the adjoint problem (2.2) is given by

$$
\begin{gathered}
-\Delta z-k^{2} z=2 k^{2} u \quad \text { in } \Omega, \\
\partial z / \partial n-\mathrm{i} k z=\mathrm{i} k u \quad \text { on } \partial \Omega .
\end{gathered}
$$

Lemma 3.2 Let $k h \leq \alpha_{0}$. Then, for the one-dimensional model problem, there holds

$$
\eta(S) \leq C_{5} C_{\mathrm{ap}} k h
$$

with $\eta$ as in (2.3). The positive constant $C_{5}$ depends only on $\alpha_{0}$. 
Proof. The exact solution to problem (3.2) in one dimension is given by

$$
z(x)=\underbrace{\mathrm{i} k \int_{-1}^{1} u(s) e^{\mathrm{i} k|s-x|} d s}_{=: z_{1}}+\underbrace{\left(-\frac{u(-1)}{2} e^{\mathrm{i} k(1+x)}-\frac{u(1)}{2} e^{\mathrm{i} k(1-x)}\right)}_{=: z_{2}} .
$$

We will approximate $z$ by interpolation. For a continuous function $u \in C^{0}(\bar{\Omega})$, the global interpolation operator $\Pi$ and its restriction $\Pi_{m}$ to an element $\tau_{m}$ are defined by

$$
\begin{array}{ll}
(\Pi u)(x):=\sum_{i=0}^{n} u_{i} b_{i}(x) & x \in \Omega, \\
\left(\Pi_{m} u\right)(x):=u_{m-1} b_{m-1}(x)+u_{m} b_{m}(x) & x \in \tau_{m},
\end{array}
$$

where the basis functions $b_{i}$ are defined as in (3.1). For the approximation of $z$ we choose $z^{\text {int }}:=\Pi z$ and estimate the error $z-z^{\text {int }}$ with respect to the $L^{2}$-norm and the $H^{1}$-seminorm. Let

$$
z_{1}^{\text {int }}:=\Pi z_{1}, \quad \text { and } \quad z_{2}^{\text {int }}:=\Pi z_{2} .
$$

From $z_{2} \in S$ we conclude that $z_{2}-z_{2}^{\text {int }}=0$ and it remains to estimate the error $z_{1}-\Pi z_{1}$. Fix an element $\tau_{m}=\left(x_{m-1}, x_{m}\right)$. For $x \in \tau_{m}=\left(x_{m-1}, x_{m}\right)$, we obtain

$$
z_{1}(x)=\underbrace{\mathrm{i} k\left\{\delta_{m-1} e^{\mathrm{i} k x}+\delta_{m} e^{-\mathrm{i} k x}\right\}}_{=: w_{1}}+\underbrace{\mathrm{i} k \int_{x_{m-1}}^{x_{m}} u(s) e^{\mathrm{i} k|s-x|} d s}_{=: w_{2}},
$$

where the numbers $\delta_{m-1}, \delta_{m}$ are defined by

$$
\delta_{m-1}:=\int_{-1}^{x_{m-1}} u(s) e^{-\mathrm{i} k s} d s \quad \text { and } \quad \delta_{m}:=\int_{x_{m}}^{1} u(s) e^{\mathrm{i} k s} d s .
$$

Let

$$
w_{1}^{\text {int }}:=\Pi_{m} w_{1} \quad \text { and } \quad w_{2}^{\text {int }}:=\Pi_{m} w_{2} .
$$

Then, $\left.z_{1}^{\text {int }}\right|_{\tau_{m}}:=w_{1}^{\text {int }}+w_{2}^{\text {int }}$. Since $w_{1}-w_{1}^{\text {int }}=0$ it remains to estimate the error $w_{2}-w_{2}^{\text {int }}$. An integration by parts yields

$$
\begin{aligned}
w_{2}(x) & =\underbrace{2 u(x)+u\left(x_{m-1}\right) e^{\mathrm{i} k\left(x-x_{m-1}\right)}+u\left(x_{m}\right) e^{\mathrm{i} k\left(x_{m}-x\right)}}_{=: v_{0}} \\
& +\underbrace{\int_{x_{m-1}}^{x} u^{\prime}(s) e^{\mathrm{i} k(x-s)} d s}_{=: v_{1}}-\underbrace{\int_{x}^{x_{m}} u^{\prime}(s) e^{\mathrm{i} k(s-x)} d s}_{=: v_{-1}}
\end{aligned}
$$

For $\mu=-1,0,1$, define $v_{\mu}^{\text {int }}:=\Pi_{m} v_{\mu}$ and observe that $v_{0}-v_{0}^{\text {int }}=0$. Hence, it remains to estimate the differences $v_{\mu}-v_{\mu}^{\text {int }}$ for $\mu= \pm 1$. The standard linear interpolation of $v_{\mu}$ is given by

$$
V_{\mu}^{\mathrm{int}}(x):=v_{\mu}\left(x_{m-1}\right) \frac{x-x_{m}}{x_{m-1}-x_{m}}+v_{\mu}\left(x_{m}\right) \frac{x-x_{m-1}}{x_{m}-x_{m-1}} .
$$

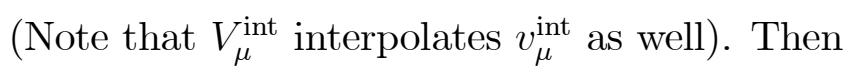

$$
v_{\mu}-v_{\mu}^{\mathrm{int}}=\left(v_{\mu}-V_{\mu}^{\mathrm{int}}\right)+\left(V_{\mu}^{\mathrm{int}}-v_{\mu}^{\mathrm{int}}\right)
$$


and we will estimate both terms separately. Standard interpolation estimates yield for $r=0,1$ the estimate

$$
\left\|\left(v_{\mu}-V_{\mu}^{\mathrm{int}}\right)^{(r)}\right\|_{L^{2}\left(\tau_{m}\right)} \leq C_{\mathrm{ap}} h_{m}^{2-r}\left|v_{\mu}^{\prime \prime}\right|_{L^{2}\left(\tau_{m}\right)} .
$$

By using $u^{\prime \prime}=-k^{2} u$, the second derivative of $v_{\mu}^{\prime \prime}$ can be written in the form

$$
v_{\mu}^{\prime \prime}=-\mu k^{2} u(x)+\mathrm{i} k u^{\prime}(x)-\mu k^{2} v_{\mu}, \quad \mu= \pm 1 .
$$

This leads to the estimate

$$
\left\|v_{\mu}^{\prime \prime}\right\|_{L^{2}\left(\tau_{m}\right)} \leq k^{2}\|u\|_{L^{2}\left(\tau_{m}\right)}+k\left\|u^{\prime}\right\|_{L^{2}\left(\tau_{m}\right)}+k^{2}\left\|v_{\mu}\right\|_{L^{2}(\tau)} .
$$

Now, a Cauchy-Schwarz inequality applied to $v_{\mu}$ in (3.4) results in

$$
\left\|v_{\mu}\right\|_{L^{2}\left(\tau_{m}\right)} \leq h_{m}\left\|u^{\prime}\right\|_{L^{2}\left(\tau_{m}\right)} .
$$

Thus, we have proved

$$
\left\|v_{\mu}^{\prime \prime}\right\|_{L^{2}\left(\tau_{m}\right)} \leq \sqrt{2} k\left(1+\alpha_{0}\right)\|u\|_{\mathcal{H}}
$$

with $\alpha_{0}$ as in the Assumptions of this lemma and this leads to

$$
\left\|\left(v_{\mu}-V_{\mu}^{\mathrm{int}}\right)^{(r)}\right\|_{L^{2}\left(\tau_{m}\right)} \leq C_{\mathrm{ap}} \sqrt{2} h_{m}^{1-r}\left(1+\alpha_{0}\right)(k h)\|u\|_{\mathcal{H}}
$$

The difference $V_{\mu}^{\text {int }}-v_{\mu}^{\text {int }}$ can be estimated by

$$
\left\|\left(V_{\mu}^{\mathrm{int}}-v_{\mu}^{\mathrm{int}}\right)^{(r)}\right\|_{L^{2}\left(\tau_{m}\right)} \leq C_{\mathrm{ap}} h^{2-r}\left\|\left(v_{\mu}^{\mathrm{int}}\right)^{\prime \prime}\right\|_{L^{2}\left(\tau_{m}\right)} .
$$

Recall

$$
v_{1}^{\text {int }}(x)=\frac{x-x_{m-1}}{x_{m}-x_{m-1}} \int_{\tau_{m}} u^{\prime}(s) e^{\mathrm{i} k(x-s)} d s .
$$

A Cauchy-Schwarz inequality leads to

$$
\left\|v_{1}^{\text {int }}\right\|_{L^{2}\left(\tau_{m}\right)} \leq \frac{h}{3}\left\|u^{\prime}\right\|_{L^{2}\left(\tau_{m}\right)} .
$$

An analogous estimate holds for $v_{-1}^{\text {int }}$. Now, $\left(v_{\mu}^{\text {int }}\right)^{\prime \prime}=-k^{2} v_{\mu}^{\text {int }}$ and we arrive at

$$
\left\|\left(v_{\mu}^{\mathrm{int}}\right)^{\prime \prime}\right\|_{L^{2}\left(\tau_{m}\right) .} \leq k(k h)\left\|u^{\prime}\right\|_{L^{2}\left(\tau_{m}\right)} .
$$

Thus,

$$
\left\|\left(V_{\mu}^{\mathrm{int}}-v_{\mu}^{\mathrm{int}}\right)^{(r)}\right\|_{L^{2}\left(\tau_{m}\right)} \leq C_{\mathrm{ap}} h^{1-r}(k h)^{2}\left\|u^{\prime}\right\|_{L^{2}\left(\tau_{m}\right)} .
$$

This leads to the final estimate

$$
\begin{aligned}
k^{1-r}\left\|\left(z-z^{\mathrm{int}}\right)^{(r)}\right\|_{L^{2}(\tau)} & \leq k^{1-r}\left\|\left(v_{\mu}-V_{\mu}^{\mathrm{int}}\right)^{(r)}\right\|_{L^{2}\left(\tau_{m}\right)}+k^{1-r}\left\|\left(V_{\mu}^{\mathrm{int}}-v_{\mu}^{\mathrm{int}}\right)^{(r)}\right\|_{L^{2}\left(\tau_{m}\right)} \\
& \leq(k h)^{2-r} C_{\text {ap }}\left(\sqrt{2}\left(1+\alpha_{0}\right)+\alpha_{0}\right)\|u\|_{\mathcal{H}} .
\end{aligned}
$$




\subsection{Estimate of $\tilde{\eta}(S)$}

In this section, we will estimate the constant $\tilde{\eta}(S)$ and recall its definition

$$
\tilde{\eta}(S)=\sup _{w \in L^{2}(\Omega) \backslash\{0\}} \inf _{v \in S} \frac{\left\|\mathcal{S}_{k} w-v\right\|_{\mathcal{H}}}{\|w\|_{L^{2}(\Omega)}}
$$

where $\mathcal{S}_{k}$ is the solution operator to the adjoint problem: For given $w \in L^{2}(\Omega)$, find $\psi \in$ $H^{1}(\Omega)$ such that

$$
a(v, \psi)=(w, v)_{L^{2}(\Omega)} \quad \forall v \in H^{1}(\Omega) .
$$

The strong formulation in one dimension is given by

$$
\begin{array}{cc}
-\psi^{\prime \prime}-k^{2} \psi=w & \text { in } \Omega, \\
-\psi^{\prime}(-1)=\mathrm{i} k \psi(-1) \wedge \psi^{\prime}(1)=\mathrm{i} k \psi(1) . &
\end{array}
$$

Lemma 3.3 Let $k h \leq \alpha_{0}$. Then, for the one-dimensional model problem, there holds

$$
\tilde{\eta}(S) \leq C_{6} C_{\mathrm{ap}} h
$$

The positive constant $C_{6}$ depends only on $\alpha_{0}$.

Proof. The exact solution of problem (3.6) is

$$
\psi(x)=i \int_{-1}^{1} w(s) \frac{e^{i k|s-x|}}{2 k} d s .
$$

Fix an element $\tau_{m}=\left(x_{m-1}, x_{m}\right)$. By using the notation as in the proof of Lemma 3.2, we get

$$
\psi=p_{1}+p_{2},
$$

where $p_{1} \in S$ and $p_{2}$ is given by

$$
p_{2}(x)=\frac{\mathrm{i}}{2 k} \int_{x_{m-1}}^{x_{m}} w(s) e^{\mathrm{i} k|s-x|} d s .
$$

Let $p_{2}^{\text {int }}:=\Pi_{m} p_{2}$. The affine interpolant of $p_{2}$ (and also of $p_{2}^{\text {int }}$ ) on $\tau_{m}$ is given by

$$
P_{2}^{\text {int }}(x)=p_{2}\left(x_{m-1}\right) \frac{x-x_{m}}{x_{m-1}-x_{m}}+p_{2}\left(x_{m}\right) \frac{x-x_{m-1}}{x_{m}-x_{m-1}} .
$$

Standard interpolation estimates show

$$
\left\|\left(p_{2}-P_{2}^{\text {int }}\right)^{(r)}\right\|_{L^{2}\left(\tau_{m}\right)} \leq C_{\mathrm{ap}} h^{2-r}\left\|p_{2}^{\prime \prime}\right\|_{L^{2}\left(\tau_{m}\right)} .
$$

The second derivative can be written in the form

$$
p_{2}^{\prime \prime}(x)=-w(x)-k^{2} p_{2}(x)
$$

and we arrive at the estimate

$$
\left\|p_{2}^{\prime \prime}\right\|_{L^{2}\left(\tau_{m}\right)} \leq\|w\|_{L^{2}\left(\tau_{m}\right)}+\frac{k h}{2}\|w\|_{L^{2}\left(\tau_{m}\right)} .
$$


Thus

$$
\left\|\left(p_{2}-P_{2}^{\text {int }}\right)^{(r)}\right\|_{L^{2}\left(\tau_{m}\right)} \leq C_{\mathrm{ap}} h^{2-r}\left(1+\alpha_{0}\right)\|w\|_{L^{2}\left(\tau_{m}\right)} .
$$

For the other term, we get

$$
\left\|\left(p_{2}^{\text {int }}-P_{2}^{\text {int }}\right)^{(r)}\right\|_{L^{2}\left(\tau_{m}\right)} \leq C_{\mathrm{ap}} h^{2-r}\left\|\left(p_{2}^{\text {int }}\right)^{\prime \prime}\right\|_{L^{2}\left(\tau_{m}\right)}
$$

and (by the same arguments as for (3.5))

$$
\left\|\left(p_{2}^{\text {int }}\right)^{\prime \prime}\right\|_{L^{2}\left(\tau_{m}\right)}=k^{2}\left\|p_{2}^{\text {int }}\right\|_{L^{2}\left(\tau_{m}\right)} \leq \frac{k h}{2}\|w\|_{L^{2}\left(\tau_{m}\right)} .
$$

Thus, we have proved

$$
k^{1-r}\left\|\left(p_{2}-p_{2}^{\text {int }}\right)^{(r)}\right\|_{L^{2}\left(\tau_{m}\right)} \leq C_{\mathrm{ap}} h(k h)^{1-r}\left(1+\frac{3}{2} \alpha_{0}\right)\|w\|_{L^{2}\left(\tau_{m}\right)}
$$

from which the assertion follows.

\subsection{Estimate of the approximation property}

It remains to estimate the infimum in (2.11) for the one-dimensional case.

Lemma 3.4 For any $f$, let $u$ be the solution of (1.2) in the one-dimensional case. Then, the interpolant $\Pi u \in S$ satisfies

$$
\|u-\Pi u\|_{\mathcal{H}} \leq C_{6} C_{\mathrm{ap}} h
$$

Proof. Let $u_{g}$ and $u_{f}$ be defined as the solutions of

$$
\begin{array}{cc}
-\Delta u_{g}-k^{2} u_{g}=0 & \text { in } \Omega, \\
\frac{\partial u_{g}}{\partial n}+\mathrm{i} k u_{g}=g & \text { on } \partial \Omega,
\end{array} \quad \text { and } \quad \begin{array}{cc}
-\Delta u_{f}-k^{2} u_{f}=f & \text { in } \Omega, \\
\frac{\partial u_{f}}{\partial n}+\mathrm{i} k u_{f}=0 & \text { on } \partial \Omega .
\end{array}
$$

Then, $u=u_{g}+u_{f}$. Note that $u_{g}$ is a linear combination of $e^{ \pm \mathrm{i} k x}$. Since $e^{ \pm \mathrm{i} k x} \in S$ the corresponding Galerkin error coincides with the exact solution $u_{g}$ and it remains to investigate

$$
\inf _{v \in S}\left\|u_{f}-v\right\|_{\mathcal{H}}
$$

The function $u_{f}$ is given by

$$
u_{f}(x)=-i \int_{-1}^{1} f(s) \frac{e^{-i k|s-x|}}{2 k} d s
$$

and coincides with (3.7) by replacing $w$ by $f$ and $k$ by $-k$ therein. Hence, we may repeat all steps of the proof of Lemma 3.3 to obtain

$$
\left\|u_{f}-u_{f}^{\mathrm{int}}\right\|_{\mathcal{H}} \leq C_{6} C_{\mathrm{ap}} h .
$$




\section{References}

[1] N. Abboud and P. Pinsky. Finite element dispersion analysis for the 3-dimensional 2nd order scalar wave-equation. Int. J. Numer. Meth. Eng., 35(6):1183-1218, 1992.

[2] M. Ainsworth. Discrete Dispersion Relation for hp-Finite Element Approximation at High Wave Number. SIAM J. Numer. Anal., to appear.

[3] I. Babuška and F. Ihlenburg. Dispersion Analysis and Error Estimation of Galerkin Finite Element Methods for the Helmholtz equation. Int. J. Numer. Meth. Eng., 38, 1995.

[4] I. Babuška and S. Sauter. Is the pollution effect of the FEM avoidable for the Helmholtz equation considering high wave numbers. SIAM, J. Numer. Anal., 34(6):2392-2423, 1997.

[5] I. M. Babuška, F. Ihlenburg, E. T. Paik, and S. A. Sauter. A Generalized Finite Element Method for Solving the Helmholtz Equation in Two Dimensions with Minimal Pollution. Comp. Meth. Appl. Mech. Eng., 128(BN-1179):325-359, 1995.

[6] A. Deraemaeker, I. Babuška, and P. Bouillard. Dispersion and pollution of the FEM solution for the Helmholtz equation in one, two and three dimesnions. Int. J. Numer. Meth. Eng., 46(4), 1999.

[7] K. Gerdes and F. Ihlenburg. On the pollution effect in finite element solutions of the 3D Helmholtz equation. Comput. Methods Appl. Mech. Engrg., 170(1), 1999.

[8] I. Harari. Reducing spurious dispersion, anisotropy and reflection in finite element analysis of time-harmonic acoustics. Comput. Methods Appl. Mech. Engrg., 140(1), 1997.

[9] I. Harari. Finite element dispersion of cylindrical and spherical acoustic waves. Comput. Methods Appl. Mech. Engrg., 190(20), 2001.

[10] I. Harari and D. Avraham. High-order finite element methods for acoustic problems. J. Comput. Acoust., 5(1), 1997.

[11] I. Harari and T. Hughes. Finite Element Methods for the Helmholtz Equation in an Exterior Domain: Model Problems. Computer Methods in Applied Mechanics and Engineering, 87:59-96, North Holland, 1991.

[12] F. Ihlenburg. Finite Element Analysis of Acousting Scattering. Springer, New York, 1998.

[13] F. Ihlenburg and I. Babuška. Finite Element Solution to the Helmholtz Equation with High Wave Number. Part I: The h-version of the FEM. Comp. Math. Appl., 39(9):9-37, 1995.

[14] F. Ihlenburg and I. Babuška. Finite Element Solution to the Helmholtz Equation with High Wave Number. Part II: The h-p version of the FEM. Siam J. Num. Anal., 34(1):315, 1997.

[15] J. M. Melenk. On Generalized Finite Element Methods. PhD thesis, University of Maryland at College Park, 1995. 
[16] A. Oberai and P. Pinsky. A numerical comparison of finite element methods for the Helmholtz equation. J. Comput. Acoust., 8(1), 2000. 